\title{
Knowledge, attitudes and practices survey of cardiac rehabilitation among cardiologists and cardiac surgeons in Lebanon
}

\author{
Rebecca Farah ${ }^{1,2^{*}}$ (D), Wim Groot ${ }^{1}$ and Milena Pavlova ${ }^{1}$
}

\begin{abstract}
Background: Cardiovascular diseases (CVDs) are among the leading causes of morbidity and mortality worldwide. Over three quarters of the cardiovascular deaths take place in low and middle-income countries. Despite the benefits, Cardiac Rehabilitation (CR) is still not routinely and not universally available. Numerous studies have found that barriers to access to $C R$ are correlated with providers, patients and environment characteristics. This first national survey on CR in Lebanon assesses the knowledge, attitudes and practices among physicians. In addition, the study identifies what the main barriers to access to $C R$ are and provides suggestions for the implementation of CR in the country.

Results: The response rate was $41.5 \%(n=83)$. Results show that the cardiologists have medium level of knowledge about CR and its multidisciplinary content. Physicians support the implementation of a comprehensive CR program in the country. $50 \%$ of the physicians recommended first to solve the financial issues before implementing a CR program. Supplementary learning about the benefits of CR is highly recommended to enroll more patients with CVD into CR. In addition, the lack of specialists in the field, lack of motivation for patients to enroll to CR and inconvenient location of the rehabilitation centers were identified as major barriers by the respondents.

Conclusions: The role of physicians in promoting patient enrolment should be optimized and exploited in the country. The access barriers identified can help to develop CR programs and to improve CR referral and enrolment rates. Funds from private parties and a budget from the government are needed to launch new $C R$ programs in the country. Further research is needed to provide evidence on the CR benefits in Lebanon and to motivate policy-makers to place priority on the establishment of a comprehensive CR program in the country.
\end{abstract}

Keywords: Cardiac rehabilitation, Prevention, Exercises, Lifestyle, Barriers, Lebanon

\section{Background}

Cardiovascular diseases (CVDs) are the number one cause of death globally: more people die annually from CVDs than from any other cause. An estimated 17.9 million people died from CVDs in 2016, representing $31 \%$ of all global deaths. Of these deaths, $85 \%$ are due to heart attack and stroke, according to the World Health

\footnotetext{
*Correspondence: r.farah@maastrichtuniversity.nl

1 Department of Health Services Research, CAPHRI, Faculty of Health, Medicine and Life Sciences, Maastricht University Medical Center;

Maastricht University, P.O. Box 616, 6200 MD Maastricht, The Netherlands

Full list of author information is available at the end of the article
}

Organization report [1] Over three quarters of the CVDs deaths take place in low- and middle-income countries $[2,3]$.

Reducing risk factors such as tobacco use (including water-pipe smoking), an unhealthy diet, obesity, and physical inactivity are the starting point to decrease the burden of CVDs in low-, middle- and high-income countries $[4,5]$. In addition, there is evidence of a positive effect of Cardiac Rehabilitation (CR) for CVDs patients [6]. CR can help to achieve a reduction in premature mortality from CVDs. A WHO report on CR in low- and middle-income countries suggests that all patients with 
CVDs should have access to CR, and healthcare providers and patients and their families should be aware of CR [7].

$\mathrm{CR}$ is defined as "a multifactorial and comprehensive non-pharmacological intervention in secondary prevention, designed to limit the [patho]physiologic and psychological effects of cardiovascular disease. CR includes components of diet, health education, advice on cardiovascular risk reduction, physical activity and stress management" [8]. CR, as a medically supervised exercise training, has the potential to act as a catalyst for promoting other facets of rehabilitation, including risk factor modification through therapeutic lifestyle changes and optimization of psychosocial support [9-14].

Evidence on the benefits of CR suggests that CR may reduce all causes of mortality by as much as $28 \%$ and may reduce cardiac mortality by up to $31 \%$ [15-20]. Also, reduced morbidity and unplanned hospital admissions in addition to improvements in exercise capacity, quality of life and psychological well-being are well-recognized CR befits reported in international guidelines [20-23].

Despite the evidence on the benefits and clinical recommendations from the American College of Cardiology and American Heart Association, CR is still not universally available in low- and middle-income countries [24, $25]$. CR is also not broadly adopted in the daily practice of physicians in high-income countries as a complementary and essential non-pharmacological intervention in the case of CVDs due to multifactorial causes [26]. Overall, CR is still frequently neglected and forgotten as part of the treatment of CVDs patients [27].

This study focuses on CR in Lebanon. Appendix 1 shows the rate of CVDs in Lebanon [2]. CVDs represent half of all deaths in Lebanon [2]. Therefore, controlling for cardiovascular risk factors is essential to improve the health of the population and to reduce the burden of CVDs. At the time of this study, in 2018, no comprehensive CR program had been implemented in Lebanon. Awareness campaigns have been implemented by the Lebanese Ministry of Public Health on CVDs and unhealthy behavior of the Lebanese population, such as a lack of physical activity, obesity, high level of smoking (for example, Lebanon ranks first in water-pipe use in the world) and a high level of CVDs deaths. Nevertheless, cardiovascular preventive programs such as $\mathrm{CR}$ are not a priority in Lebanon.

The objective of this study is to identify the knowledge, attitudes and practices of Lebanese cardiologists about CR. In particular, this study assesses the discharge treatment recommendations for patients within CVDs in Lebanon. Key barriers faced by cardiologists and cardio-surgeons to refer patients to $\mathrm{CR}$ are also identified. This study is descriptive and is based on the Knowledge,
Attitude and Practice (KAP) framework. The study also provides suggestions for the future development of CR and takes into consideration the recommendations of cardiologists and cardiac surgeons for further expansion of CR practice.

\section{Methods}

A quantitative cross-sectional design was adopted using a structured, self-administrated KAP questionnaire. The survey was conducted during the $13^{\text {th }}$ International conference of the Lebanese Cardiology Society (LCS) \& the 4th Middle East Heart Failure Meeting held in December 2018, in Beirut, Lebanon. The participants were cardiologists and cardiac surgeons in Lebanon. Ethical approval to conduct this survey was obtained from the President of the LCS, and from the ethical committee of the society.

All medical doctors who attended the conference were invited to fill in the questionnaire. These included both Lebanese and foreign medical doctors. No selection of respondents was made. All conference participants could take the survey. The participants did not receive any incentive to take part in the study. The questionnaire was distributed by hand by the LCS board and the filledin questionnaires were collected by the organizers of the 3-day LCS conference. In total, 83 out of 200 conference participants returned a filled-in questionnaire.

The self-administrated questionnaire was designed based on a literature review. This questionnaire was validated by experts in the field, such as cardiologists from the LCS. The questionnaire was updated according to their suggestions. The questionnaire was based on the three components of the KAP framework of the WHO (27) and contained 25 questions. We added two components: one about barriers faced by cardiologists and one about the socio-demographic data of the respondents. Appendix 2 shows the 25 questions asked to the physicians.

The questionnaire was divided into 5 parts:

- Part 1 covered questions on knowledge of physicians about CR

- Part 2 explored the attitudes of physicians on CR

- Part 3 assessed the daily practices of physicians on CR

- Part 4 investigated the barriers faced by physicians when referrer patients to $\mathrm{CR}$

- Part 5 studied the socio-demographic data of the responders.

The questionnaire was anonymous. Written informed consent was obtained from each participant at the beginning of the questionnaire. The wording of 
questions was pre-tested before the survey. The English version of the questionnaire was used in the survey.

After the data collection and screening, data entry, cleaning and analysis were done by using the software package SPSS version 24. The analysis consisted of descriptive statistics, namely frequencies, mean, median, standard deviation, percentage for each response variable.

\section{Results}

In total, 83 questionnaires were gathered among the 200 participants in the conference. Thus, the overall response rate was $41.5 \%$. Results are shown in the tables below.

\section{Socio-demographic characteristics}

Table 1 shows that $57.7 \%$ of the physicians who participated in the study were cardiologists. More than half of the participants $(62.3 \%)$ had graduated in Lebanon. Respondents were for $76.6 \%$ males and $23.4 \%$

Table 1 Socio-demographic characteristics of the respondents

\begin{tabular}{|c|c|c|c|c|c|}
\hline \multirow[t]{6}{*}{ Age } & $<35$ & N (\%) & $22(28.6 \%)$ & Mean & 2.4 \\
\hline & $35-40$ & N (\%) & 19 (24.7\%) & Median & 2 \\
\hline & $40-55$ & N (\%) & $23(29.9 \%)$ & SD & 1.17 \\
\hline & $55-65$ & N (\%) & $9(11.7 \%)$ & & \\
\hline & $>65$ & N (\%) & $4(5.2 \%)$ & & \\
\hline & & $N$ total & 77 & & \\
\hline \multirow[t]{3}{*}{ Gender } & Male & N (\%) & $59(76.6 \%)$ & Mean & 1.8 \\
\hline & Female & N (\%) & $18(23.4 \%)$ & Median & 2 \\
\hline & & $N$ total & 77 & SD & 0.487 \\
\hline \multirow[t]{7}{*}{ Function } & Cardiologist & N (\%) & 45 (57.7\%) & Mean & 2.17 \\
\hline & Cardiac surgeon & N (\%) & $8(10.3 \%)$ & Median & 1 \\
\hline & Manager & N (\%) & $4(5.1 \%)$ & SD & 1.6 \\
\hline & Cardiology fellow & N (\%) & $9(11.5 \%)$ & & \\
\hline & $\mathrm{MD}$ & N (\%) & $11(14.1 \%)$ & & \\
\hline & Others & N (\%) & $1(1.3 \%)$ & & \\
\hline & & $N$ total & 78 & & \\
\hline \multirow[t]{5}{*}{ Education } & Lebanon & N (\%) & $33(53.2 \%)$ & Mean & 1.85 \\
\hline & US & N (\%) & $5(8.1 \%)$ & Median & 1 \\
\hline & EU & N (\%) & $19(30.6 \%)$ & SD & 1.16 \\
\hline & Others & N (\%) & $5(8.1 \%)$ & & \\
\hline & & $\mathrm{N}$ total & 62 & & \\
\hline \multirow[t]{4}{*}{ Work Location } & Rural & N (\%) & 27 (37\%) & Mean & 1.65 \\
\hline & Urban & N (\%) & $44(60.3 \%)$ & Median & 2 \\
\hline & Both & N (\%) & $2(2.7 \%)$ & SD & 0.532 \\
\hline & & $\mathrm{N}$ total & 73 & & \\
\hline \multirow[t]{5}{*}{ Place of graduation } & Lebanon & N (\%) & $48(62.3 \%)$ & Mean & 1.85 \\
\hline & US & N (\%) & $2(2.6 \%)$ & Median & 1 \\
\hline & EU & N (\%) & $17(22.1 \%)$ & SD & 1.16 \\
\hline & Others & N (\%) & $10(13 \%)$ & & \\
\hline & & $N$ total & 77 & & \\
\hline \multirow[t]{4}{*}{ Work location office } & Hospital settings & N (\%) & $49(66.2 \%)$ & Mean & 1.59 \\
\hline & Private office & N (\%) & $6(8.1 \%)$ & Median & 1 \\
\hline & Both & N (\%) & $19(25.7 \%)$ & SD & 0.87 \\
\hline & & $\mathrm{N}$ total & 74 & & \\
\hline \multirow[t]{5}{*}{ Number of Years of practice } & $<5$ & N (\%) & $22(28.6 \%)$ & Mean & 2.59 \\
\hline & 10-May & $\mathrm{N}(\%)$ & $12(15.6 \%)$ & Median & 3 \\
\hline & $15-O c t$ & $\mathrm{~N}(\%)$ & $42(54.5 \%)$ & SD & 1.22 \\
\hline & $>15$ & $N(\%$ & $1(1.3 \%)$ & & \\
\hline & & N total & 77 & & \\
\hline
\end{tabular}


were females. In total, $29.9 \%$ of the participants were $40-50$ years old and $28.6 \%$ were younger than 35 . In addition, more than half of the participants (54.5\%) had 10 to 15 years of practice experience. In total, $60.3 \%$

Table 2 Knowledge about cardiac rehabilitation (CR) among the respondents

\begin{tabular}{|c|c|c|c|c|c|}
\hline \multirow{6}{*}{$\begin{array}{l}\text { Knowledge } \\
\text { about CR- } \\
\text { level }\end{array}$} & Very poor & N (\%) & $4(4.8 \%)$ & Mean & 3.28 \\
\hline & Poor & N (\%) & 14 (16.9\%) & Median & 3 \\
\hline & Medium & N (\%) & 29 (39.9\%) & SD & 1.04 \\
\hline & Good & N (\%) & $26(31.3 \%)$ & & \\
\hline & Excellent & N (\%) & $10(12 \%)$ & & \\
\hline & & N Total & 83 & & \\
\hline \multirow{6}{*}{$\begin{array}{l}\text { Knowledge } \\
\text { about CR- } \\
\text { content }\end{array}$} & Very poor & N (\%) & 9 (10.8\%) & Mean & 2.93 \\
\hline & Poor & N (\%) & $22(26.5 \%)$ & Median & 3 \\
\hline & Medium & N (\%) & $26(31.3 \%)$ & SD & 1.16 \\
\hline & Good & N (\%) & 17 (20.5\%) & & \\
\hline & Excellent & N (\%) & $9(10.8 \%)$ & & \\
\hline & & N Total & 83 & & \\
\hline
\end{tabular}

of the participants practiced in urban areas and $66.2 \%$ practiced in a hospital.

\section{Knowledge about CR}

Table 2 presents the results on the level of knowledge about CR. The table shows that almost one-third (31.3\%, $n=26)$ of the participants stated that they had good knowledge of CR and $4.8 \%(n=4)$ said they had very poor knowledge of CR. Regarding the content of the program, $31.3 \%$ of participants $(n=26)$ stated they had a medium level of knowledge of the multidisciplinary components of CR and $10.8 \%(n=9)$ had very poor knowledge of the multidisciplinary CR components.

\section{Attitude towards CR}

Table 3 depicts the results on attitudes towards CR. More than half of the physicians in our study $(55.4 \%, n=46)$ agreed that a patient who is stable after Acute Coronary Syndrome could be enrolled into CR. Half of the participants $(51.8 \%, n=43)$ thought that CR in Lebanon could be effective. Less than half of responders $(53.2 \%, n=33)$ considered that access to an outpatient CR center could have an added value. Overall, $52.4 \%(n=43)$ of the physicians in our sample strongly agreed that quality of the treatment would increase for patients who have received

Table 3 Attitude toward cardiac rehabilitation (CR) among the respondents

\begin{tabular}{|c|c|c|c|c|c|}
\hline \multirow[t]{6}{*}{ Do you think that a patient is stable post ACS could be enrolled into a CR? Please rate: } & Strongly agree & N (\%) & $23(27.7 \%)$ & Mean & 1.95 \\
\hline & Agree & $N(\%)$ & $46(55.4 \%)$ & Median & 2 \\
\hline & Neutral & N (\%) & $10(12.0 \%)$ & SD & 0.81 \\
\hline & Disagree & N (\%) & $3(3.6 \%)$ & & \\
\hline & Strongly disagree & N (\%) & $1(1.2 \%)$ & & \\
\hline & & N Total & 83 & & \\
\hline \multirow[t]{6}{*}{ Do you think that CR in Lebanon could be effective? } & Strongly agree & $N(\%)$ & $24(28.9 \%)$ & Mean & 1.927 \\
\hline & Agree & $N(\%)$ & $43(51.8 \%)$ & Median & 2 \\
\hline & Neutral & $N(\%)$ & $14(16.9 \%)$ & SD & 0.745 \\
\hline & Disagree & N (\%) & $2(2.4 \%)$ & & \\
\hline & Strongly disagree & $N(\%)$ & 0 & & \\
\hline & & N Total & 83 & & \\
\hline \multirow[t]{6}{*}{ Do you consider that access for an outpatient CR center is an added value in the country? } & Strongly agree & N (\%) & $34(41 \%)$ & Mean & 1.734 \\
\hline & Agree & N (\%) & $38(45.8 \%)$ & Median & 2 \\
\hline & Neutral & N (\%) & $10(12.0 \%)$ & SD & 0.717 \\
\hline & Disagree & N (\%) & $1(1.2 \%)$ & & \\
\hline & Strongly disagree & N (\%) & 0 & & \\
\hline & & N Total & 83 & & \\
\hline \multirow[t]{6}{*}{ Do you think that the outcome will be improved if your patients are enrolled in a CR } & Strongly agree & N (\%) & $43(52.4 \%)$ & Mean & 1.6 \\
\hline & Agree & N (\%) & $28(34.1 \%)$ & Median & 1 \\
\hline & Neutral & N (\%) & $11(13.4 \%)$ & SD & 0.715 \\
\hline & Disagree & N (\%) & 0 & & \\
\hline & Strongly disagree & N (\%) & 0 & & \\
\hline & & N Total & 82 & & \\
\hline
\end{tabular}


CR. Table 3: Attitude of cardiologists towards CR $(n=83)$.

\section{Practice of $C R$ referrals}

Table 4 presents the results on the practice regarding CR. The table shows that more than half of the physicians in our study (53\%) would refer patients to CR after discharge from the hospital. Results show that $36.1 \%$ $(n=30)$ of the cardiologists consider "Heart Failure (HF) patients" as the main type of patient to be referred to CR. The second type of patients to be referred for CR are post valve surgery patients $(26 \% n=22)$. More than half of the participants in the study, $65.1 \%(n=54)$, stated that it would be difficult to refer patients to CR in Lebanon. In total, $73.8 \%(n=59)$ of the participants think that action must be taken by insurance companies, professional health givers, physicians, policy providers and the Ministry of Health to introduce CR programs in the country. Only $3.9 \%(n=3)$ stated that they would never refer a patient to a CR program if such program would become available in the future and $48.7 \%$ (37) indicated that they already sent patients to CR 3-5 times per month. After discharging the patient from hospital, 48.1\% $(n=38)$ of physicians in our study ask their patients to start a

Table 4 Practice of cardiac rehabilitation (CR) by the respondents

\begin{tabular}{|c|c|c|c|c|c|}
\hline \multirow[t]{7}{*}{ When would you refer a patient to start a CR? } & Starting in the hospital settings & $\mathrm{N}(\%)$ & $23(27.7 \%)$ & Mean & 1.92 \\
\hline & Directly after the hospital discharge & & & Median & 2 \\
\hline & 4 weeks or more after the discharge & $\mathrm{N}(\%)$ & $44(53.0 \%)$ & SD & 0.711 \\
\hline & Would not refer & & & & \\
\hline & & N (\%) & $15(18.1 \%)$ & & \\
\hline & & $\mathrm{N}(\%)$ & $1(1.2 \%)$ & & \\
\hline & & NTotal & 83 & & \\
\hline \multirow{7}{*}{$\begin{array}{l}\text { What kind of patients do you consider suitable for CR after } \\
\text { discharging from hospital? }\end{array}$} & HF/CAD/Angina/ACS & $\mathrm{N}(\%)$ & $30(36.1 \%)$ & Mean & 3.5 \\
\hline & $\mathrm{CABG}$ & $\mathrm{N}(\%)$ & $10(12 \%)$ & Median & 3 \\
\hline & Post valve surgery & $\mathrm{N}(\%)$ & $2(2.4 \%)$ & SD & 2.43 \\
\hline & Pacemaker & $\mathrm{N}(\%)$ & $22(26.5 \%)$ & & \\
\hline & Post vascular surgery & $N(\%)$ & $1(1.2)$ & & \\
\hline & All of the above & $N(\%)$ & $18(21.7 \%)$ & & \\
\hline & & N Total & 83 & & \\
\hline \multirow[t]{3}{*}{ Would it be difficult to refer patients to CR in the country? } & Yes & $N(\%)$ & $54(65.1 \%)$ & MEAN & 1.4 \\
\hline & No & $\mathrm{N}(\%)$ & $29(34.9 \%)$ & Median & 1 \\
\hline & & NTotal & 83 & SD & 0.644 \\
\hline \multirow[t]{6}{*}{ Who should take initiative to initiate CR in the country? } & Insurance companies & $\mathrm{N}(\%)$ & $5(6.3 \%)$ & Mean & 4.31 \\
\hline & Professional care givers & $\mathrm{N}(\%)$ & $6(7.5 \%)$ & Median & 5 \\
\hline & Physicians & $N(\%)$ & $7(8.8 \%)$ & $\mathrm{SD}$ & 1.26 \\
\hline & Policy makers & $\mathrm{N}(\%)$ & $3(3.8 \%)$ & & \\
\hline & Ministry of Health & $\mathrm{N}(\%)$ & $59(73.8 \%)$ & & \\
\hline & & NTotal & 80 & & \\
\hline \multirow{6}{*}{$\begin{array}{l}\text { How frequently would you send patients to CR if CR would } \\
\text { have started? }\end{array}$} & $3-5$ times per month & $\mathrm{N}(\%)$ & $37(48.7 \%)$ & Mean & 1.85 \\
\hline & 3-5 times per week & $N(\%)$ & $21(27.6 \%)$ & Median & 2 \\
\hline & $1-2$ times per month & $N(\%)$ & $13(17.1 \%)$ & $\mathrm{SD}$ & 1.05 \\
\hline & Once every 6 months & $N(\%)$ & $2(2.6 \%)$ & & \\
\hline & Never & $N(\%)$ & $3(3.9 \%)$ & & \\
\hline & & NTotal & 76 & & \\
\hline \multirow[t]{6}{*}{ After discharge physicians asked patients to: } & To do nothing, to be at rest & $N(\%)$ & $1(1.3 \%)$ & Mean & 3.59 \\
\hline & To exercise a bit & N (\%) & 28 (35.4\%) & Median & 3 \\
\hline & To go to fitness club & $N(\%)$ & $11(13.9 \%)$ & SD & 1.41 \\
\hline & To take it easy & $\mathrm{N}(\%)$ & $1(1.3 \%)$ & & \\
\hline & To start a rehab program & $\mathrm{N}(\%)$ & $38(48.1 \%)$ & & \\
\hline & & NTotal & 79 & & \\
\hline
\end{tabular}


rehabilitation program. In total, $35.4 \%$ of the respondents request their patients to exercise a bit and $1.3 \%$ recommend to their patients to get bedrest. Table 4: Practice of cardiologist regarding $\mathrm{CR}(N=83)$.

\section{Barriers related to $C R$}

In Table 5, the results on barriers faced by physicians are presented. It shows that nearly all participants in our study $(77.7 \%, n=59)$ face barriers to refer patients to CR. Results presented in Table 5 demonstrate the kind of barriers that physicians face. The major barriers are a lack of specialists in the field, followed by a lack of motivation for the patient to enroll in CR and the location of the center.

\section{Support for CR}

Results shown in Table 6 indicate that $84.6 \%(n=66)$ of the participants in our study will fully support an inpatient CR program, $96.2 \%(n=75)$ will support a future outpatient CR and $85.9 \%(n=67)$ will support homebased cardiac tele-rehabilitation. Table 6: Support from Physicians to all kinds of CR $(N=83)$.

\section{Respondents' recommendations for CR}

Half of the respondents (50\%) recommended that first the financial issues need to be solved before implementing a CR program in Lebanon, and second that CR needs to be covered by insurance and government policies. The third recommendation by more than a quarter of the participants was related to additional education for medical doctors and public awareness about CR.

Table 5 Barriers to access cardiac rehabilitation (CR) according to the respondents

\begin{tabular}{|c|c|c|c|c|c|}
\hline \multirow{3}{*}{$\begin{array}{l}\text { Existence of } \\
\text { barriers to refer } \\
\text { patients to } \\
\text { the CR }\end{array}$} & Yes & N (\%) & $59(77.6 \%)$ & Mean & 1.34 \\
\hline & No & N (\%) & $17(22.4 \%)$ & Median & 1 \\
\hline & & N Total & 76 & SD & 1.19 \\
\hline \multirow{7}{*}{$\begin{array}{l}\text { Kind of barriers } \\
\text { faced by physi- } \\
\text { cians }\end{array}$} & Lack of specialists & N (\%) & $18(23.7 \%)$ & Mean & 5.14 \\
\hline & Lack of knowledge & N (\%) & $3(3.9 \%)$ & Median & 7 \\
\hline & Lack of motivation & N (\%) & $4(5.3 \%)$ & SD & 2.54 \\
\hline & Cost of care & N (\%) & $1(1.3 \%)$ & & \\
\hline & $\begin{array}{l}\text { Localization of the } \\
\text { center }\end{array}$ & N (\%) & $4(5.3 \%)$ & & \\
\hline & All of the above & $\mathrm{N}(\%)$ & $46(60.5 \%)$ & & \\
\hline & & N Total & 76 & & \\
\hline
\end{tabular}

Table 6 Support for Cardiac Rehabilitation (CR) among to the respondents

\begin{tabular}{llllll}
\hline Support for inpatient CR & Yes & N (\%) & $66(84.6 \%)$ & Mean & 1.15 \\
& No & N (\%) & $12(15.4 \%)$ & Median & 1 \\
& & N Total & 78 & SD & 0.363 \\
Support for outpatient CR & Yes & N (\%) & $75(96.2 \%)$ & Mean & 1.03 \\
& No & N (\%) & $3(3.8 \%)$ & Median & 1 \\
& & N Total & 78 & SD & 0.19 \\
$\begin{array}{l}\text { Support for home-based } \\
\text { cardiac tele- rehabilitation }\end{array}$ & Yes & N (\%) & $67(85.9 \%)$ & Mean & 1.16 \\
& No & N (\%) & $11(14.1 \%)$ & Median & 1 \\
& & N Total & 78 & SD & 0.467 \\
\hline
\end{tabular}

\section{Discussion}

This study is the first to assess CR in Lebanon. Our findings show that physicians have some knowledge about $\mathrm{CR}$ and its components despite the absence of comprehensive CR programs in the country. The majority of the respondents strongly approved of $\mathrm{CR}$ and recognized its added value, as well as the beneficial CR outcomes. Regarding their practice, nearly all participants responded that it is difficult to refer a patient to $C R$. They expect to face barriers if a comprehensive $C R$ program is implemented in the near future in Lebanon. Therefore, it was relevant to identify what kind of barriers respondents considered relevant in the case of CR, as well as what their suggestions and recommendation were for the further development of CR programs.

Our results found a medium level of knowledge of providers about CR. Several studies conducted in other countries on this topic, have found a correlation between knowledge and health education. For example, one study found that a lack of knowledge on CR is due to a deficiency of health education among both inpatient and outpatient providers, specifically on the importance of CR in improving outcomes in cardiac patients [36, 37]. The main barriers to CR referrals found in our study, according to the respondents, were the lack of specialists in the country, followed by a lack of motivation of the patient to enroll in a program and the location of the center, which is also an important factor. As suggested in the literature [37], despite the evidence on CR, the rate of patients' enrollment in CR is very low, and even in highincome countries, cardiologists referred only 20 to $30 \%$ of their patients.

According to one study conducted on global access to $\mathrm{CR}$, worldwide, there is low availability of CR; only $38.8 \%$ of countries globally have CR programs. Specifically, $68.0 \%$ of high-income and $23 \%$ of low- and middle-income countries $(28.2 \%$ for middle- and $8.3 \%$ for low-income countries) have CR. CR density estimates 
ranged from 1 program per 0.1-6.4 million inhabitants [27]. In the USA, for example, participation rates range from 14 to $55 \%$ after myocardial infarction; in the UK, $28,6 \%$ of eligible patients were enrolled in CR; in Canada, about $30 \%$ of cardiac inpatients enroll in CR [6]. The reasons why cardiac patients are not participating in CR programs, are multifactorial and are related to a nonpowerful enrollment after the referrals, no motivation of the patients, lack of insurance coverage of $\mathrm{CR}$ and inconvenient location of the $\mathrm{CR}$ center. A study conducted in Canada found that more knowledge and familiarity with $\mathrm{CR}$ guidelines among healthcare providers, is associated with higher self-reported referral rates [28].

Moreover, our findings showed that one of the key barriers to CR referrals was a lack of motivation of the patients to adhere to a program, as stated by respondents in our study. Several studies on providers barriers to refer patients to CR [36] have demonstrated that physicians' attitude and behavior are key factors affecting patient's adherence to the program. According to one systematic review, the strength of the primary care physician recommendations is highly correlated with patients' adherence to CR [29]. One study published in the Journal of the American College of Cardiology in 2009, found that the strongest determinant of patient participation in $\mathrm{CR}$ is the strength of the recommendation of the physician making the referral. If the physician believes and conveys the importance "passionately", patients are more likely to participate [30].

One study on physician attitudes toward CR in the Philippines has shown that lower CR enrollment is correlated with cardiologists who are more uncertain about the benefits of a CR program [29]. As reported in a systematic literature review, a large study conducted on 22666 cardiac inpatients eligible for $\mathrm{CR}$, found that patients who enrolled in a program received strong physician support to start CR, whereas those who did not enroll received either a weak or no recommendation at all [31]. Other studies found that medical specialties play an important role in the referral to CR [30]. Generalists were less likely to referrer patients to CR compared with specialists, such as cardiologists or cardiac surgeons at the discharge time. Additionally, the literature suggests that it will be better for patients to be seen by a specialist during their hospitalization and to be referred to CR prior to their hospital discharge to increase the level of participation in a CR program $[32,33]$. The procedure is important for the enrollment of the patient to CR. A study reported referral rates of $48 \%$ among patients with percutaneous coronary intervention and $91 \%$ of patients who underwent cardiac surgery. The studies found that the strongest predictor of CR referral was the hospital performing the procedure [34].
Our study has also explored the recommendations of participants for the further development of CR in Lebanon. Results showed that there is a need to solve the financial issue of covering patients who enroll in CR and a need to change the Lebanese healthcare system for this. The healthcare system in Lebanon is funded more through private than public resources, and it has been overstretched by the increase in refugees from neighboring countries. No national strategic plan is available for the implementation of $C R$ centers, and no budget is given from the government for CR. Our results demonstrate that physicians support all forms of $\mathrm{CR}$, including inpatient, outpatient and home-based programs. Likewise, respondents in our study suggested more health education for providers and public awareness of CR benefits.

After the survey was conducted in 2019, two CR programs have opened in two hospitals in Lebanon. One in Beirut West and another one in Mount-Lebanon area, which will facilitate the access for patients suffering from CVD in the Beirut district. As suggested, barriers correlated to physicians' attitude and practice need to be overcome by promoting the benefits of the programs among physicians in order to have more powerful and enthusiastic endorsement after discharge of the patients. Also, another study suggested that a "motivational letter" provided to the patients can be helpful to remind the physicians to initiate a CR referral 35 . To have a strong endorsement in every medical establishment, CR must be in the procedure of the treatment for every CVD and must be cover by insurance and government.

\section{Limitations}

Our study has some limitations. The survey was only done in English and not in Arabic or French. Although we recognize this limitation, we do not expect that it had a substantial influence on the results because the participants were professionals in the field of the study able to communicate in English. Nevertheless, some of the questions might have seemed hypothetical to the respondents since CR is largely absent in Lebanon. It is unclear how actual experience with referrals to $C R$ in Lebanon would have influenced their responses. Another limitation was the difficulty to properly read the suggestions and comments of three respondents due to unclear handwriting. Also, we cannot exclude self-selection bias. Specifically, participants in the survey might have been more interested in CR and more positive about CR than the average medical doctor. This might have skewed the results toward positive opinions. The participants were a heterogeneous group, e.g. only about half of them were actually practicing in Lebanon and about two thirds were practicing CT surgeons or cardiologists. Moreover, the 
response rate is not very high, which limits the possibility to extrapolate our findings to the entire study population.

\section{Conclusions}

As indicated by the study results and their discussion, Lebanese healthcare providers need more information and training on $\mathrm{CR}$, which suggests that such education programs are important to develop. Supplementary learning about the benefits of CR is highly recommended to enroll more patients with CVD into CR. It is important to integrate $\mathrm{CR}$ in the procedure of CVD management in every hospital in Lebanon. There is also a need to provide coverage of CR in Lebanon. A significant endorsement of CR by a specialist at the hospital discharge, with a convincing explanation of CR benefits to the patients, is crucial to have a higher enrollment of patients to the new $C R$ programs. By ensuring that $C R$ is available to heart patients and expenses are covered, a reduction in cardiac morbidity and mortality can be expected at a national level, which is the top chronic disease problem in the country. Finally, the financial barrier needs to be overcome with a call for funding and further researches to provide evidence for policy.

\section{Key message of this study What is already known on this subject?}

- Benefits of CR, providers barriers to enroll in a CR

\section{What might this study add?}

- First study assessing CR in Lebanon among physicians

- Suggestions for implementation of CR in the country

- Local barriers identified and how to overcome

- Need of solving the financial issue to cover CR

- Need to have a policy from the government

\section{How might this impact on clinical practice?}

- Need of a good communication between physicians and patients

- Timing of the communication and by a specialist must be consider

- $\mathrm{CR}$ need to be routinely prescribed, be inside the procedure of the management of the CVD

- Importance to enroll in a CR for burden in CVD in 2030

- To change bad habits of patients

- Suggestion for CR to reduce CVD death in Lebanon

\section{Abbreviations}

CVDs: Cardiovascular diseases; CR: Cardiac Rehabilitation; WHO: World Health Organization; SPSS: Statistical Package of Social Sciences; KAP: Knowledge Attitude and Practice; LCS: Lebanese Cardiology Society.

\section{Supplementary Information}

The online version contains supplementary material available at https://doi. org/10.1186/s43044-021-00212-2.

Additional file 1. Timing of communication by a specialist needs to be considered.

\section{Acknowledgements}

Authors would like to thank the Lebanese Cardiology Society to give ethical approval to conduct the survey during the annual congress. Only the abstract of the paper was selected and presented through ePoster at the European Association of Preventive Cardiology.

\section{Authors' contributions}

Design of the study: RF, MP, WG. Acquisition of data: RF. Statistical analysis of data: RF, MP, WG. Interpretation of data: RF, MP, WG. Draft the work: RF. Revision of the work: RF, MP,WG. All of the authors have read and approved the manuscript.

Funding

All Authors have no disclosure of findings.

Availability of data and material

Data are stored in Maastricht University and are available upon request if needed.

\section{Declarations}

Ethics approval and consent to participate

Ethical approval was obtained from Maastricht University ethical board and from the Lebanese Cardiology Society. The ethical committee number is not applicable. Every respondent gave a written consent to participate in this research.

\section{Consent for publication}

All participants gave their consent for publication.

Competing interests

All Authors have no conflicts of interest.

\section{Author details}

${ }^{1}$ Department of Health Services Research, CAPHRI, Faculty of Health, Medicine and Life Sciences, Maastricht University Medical Center; Maastricht University, P.O. Box 616, 6200 MD Maastricht, The Netherlands. ${ }^{2}$ Department of Rehabilitation and Physical Therapy (Group A); Delta-Chirec Hospitals Group, Brussels, Belgium.

Received: 22 July 2021 Accepted: 11 September 2021

Published online: 14 October 2021

References

1. World Health Organization. Report on cardiovascular diseases (CVDs). 17 May 2017.https://www.who.int/news-room/fact-sheets/detail/cardiovasc ular-diseases-(cvds).

2. World Health Organization report on CVDs in Lebanon. Noncommunicable diseases (NCD) Country Profiles, 2014.https://www.who.int/nmh/ countries/lbn_en.pdf 
3. Deek H, Newton P, Inglis S et al (2015) Heart Health in Lebanon and considerations for addressing the burden of cardiovascular disease. Collegian 22(3):333-339

4. World health organization country factsheet report 2018. Insufficient Physical activity. https://www.who.int/news-room/fact-sheets/detail/physi cal-activity

5. Al Thannir M, Kobrosly S, Itani T, El-Rajab M, Tannir S. Prevalence of physical activity among lebanese adults: a cross-sectional study. J Phys Activity Health, 2009 May;6(3),315-320. https://www.ncbi.n/m.nih.gov/pubmed/ 19564659

6. Dalal HM, Doherty P, Taylor RS. Clinical review on cardiac rehabilitation. BMJ 2015:351:h5000

7. Grace SL et al (2016) Cardiac delivery model in low-resource settings. Heart. https://doi.org/10.1136/heartjnl-2015-309209

8. Singh VH. Cardiac Rehabilitation. The heart.org Medscape, 2015 august.

9. British Association for Cardiovascular Prevention and Rehabilitation. BACPR standards and core components for cardiovascular disease prevention and rehabilitation 2017.2 ${ }^{\text {nd }}$ ed. UKBACPR, 2017. http://www.bacpr.com/resou rces/AC6_BACPRStandards\&CoreComponents2017.pdf

10. National Institute for Health and Care Excellence. Secondary prevention in primary and secondary care for patients following a myocardial infarction (clinical guidance 172). NICE, 2013. https://www.nice.org.uk/guidance/ cg172/evidence/myocardial-infarction-secondary-prevention-full-guide line-pdf-248682925

11. JBS3 Board (2014) Joint British Societies' consensus recommendations for the prevention of cardiovascular disease (JBS3). Heart 100(suppl2):i1-67. https://doi.org/10.1136/heartjnl-2014-305693

12. Piepoli MF, Corrà U, Adamopoulos S, Benzer W, Bjarnason-Wehrens B, Cupples M, et al; Endorsed by the Committee for Practice Guidelines of the European Society of Cardiology. Secondary prevention in the clinical management of patients with cardiovascular diseases. Core components, standards and outcome measures for referral and delivery: a policy statement from the cardiac rehabilitation section of the European Association for Cardiovascular Prevention \& Rehabilitation. Eur J Prev Cardiol 2014; 21:664-81.

13. Balady GJ, Williams MA, Ades PA, Bittner V, Comoss P, Foody JA, et al; American Heart Association Exercise, Cardiac Rehabilitation, and Prevention Committee; Council on Clinical Cardiology; Councils on Cardiovascular Nursing, Epidemiology and Prevention, and Nutrition, Physical Activity, and Metabolism; American Association of Cardiovascular and Pulmonary Rehabilitation. Core components of cardiac rehabilitation/secondary prevention programs: 2007 update: a scientific statement from the American Heart Association Exercise, Cardiac Rehabilitation, and Prevention Committee, the Council on Clinical Cardiology; the Councils on Cardiovascular Nursing, Epidemiology and Prevention, and Nutrition, Physical Activity, and Metabolism; and the American Association of Cardiovascular and Pulmonary Rehabilitation. J Cardiopulm Rehabil Prev:2007; 27:121-9.

14. Leon AS, Franklin BA, Costa F, Balady GJ, Berra KA, Stewart KJ, et al; American Heart Association; Council on Clinical Cardiology (Subcommittee on Exercise, Cardiac Rehabilitation, and Prevention); Council on Nutrition, Physical Activity, and Metabolism (Subcommittee on Physical Activity); American association of Cardiovascular and Pulmonary Rehabilitation. Cardiac rehabilitation and secondary prevention of coronary heart disease: an American Heart Association scientific statement from the Council on Clinical Cardiology (Subcommittee on Exercise, Cardiac Rehabilitation, and Prevention) and the Council on Nutrition, Physical Activity, and Metabolism (Subcommittee on Physical Activity), in collaboration with the American association of Cardiovascular and Pulmonary Rehabilitation. Circulation.2005; 111:369-76.

15. Taylor RS, Brown A, Ebrahim S et al (2004) Exercise-Based rehabilitation for Patients with coronary heart disease: systematic review and meta-analysis of randomized controlled trial. Am J Med 116:682-692

16. Oldrige N, Guyatt G, Fisher M. Cardiac rehabilitation after myocardiac infaction:combined xperience of randomized clinical trials. JAMA.1998:260:945-950

17. Taylor R, Brown A, Ebrahim S, Jollife J, Noorani H, Rees K, Skidmore B, Stone J, Thompson D, Oldrige N (2004) exercise-based rehabilitation for patients with coronary heart disease: systematic review and meta-analysis of randomized controlled trials. Am J Med 116:682-692

18. Jollife J, Rees K, Taylor R, Thompson D, Oldrige N, Ebrahim S. Exercise-based rehabilitation for coronary heart disease (Cocrane review). Cochrane Database Syst Rev.2001;(1):CD001800

19. Heran BS, Chen JM, Ebrahim S, Moxham T, Oldridge N, Rees K, et al. Exercisebased cardiac rehabilitation for coronary heart disease. Cochrane Database Syst Rev2011;(7):CD001800.

20. West RR, Jones DA, Henderson AH (2012) Rehabilitation after myocardial infarction trial (RAMIT): multi-centre randomised controlled trial of comprehensive cardiac rehabilitation in patients following acute myocardial infarction. Heart 98:637-644

21. Doherty P, Lewin R (2012) The RAMIT trial, a pragmatic RCT of cardiac rehabilitation versus usual care: what does it tell us? Heart 98:605-606

22. Lawler PR, Filion KB, Eisenberg MJ (2011) Efficacy of exercise-based cardiac rehabilitation post-myocardial infarction: a systematic review and metaanalysis of randomized controlled trials. Am Heart J 162:571-584.e2

23. Wood D (2012) Is cardiac rehabilitation fit for purpose in the NHS: maybe not. Heart 98:6078

24. Thomas RJ et al. ACC/AHA Clinical Performance and Quality Measure for Cardiac Rehabilitation. A report of the American College of Cardiology/ American Heart Association. Task Force on Performance Measures. J Am Coll Cardiol. 2018 April;71(16).

25. Burns P, Rohrich R, Chung K (2011) The levels of evidence and their role in evidence-based medicine. Plast Reconstr Surg 128(1):305-310

26. Piepoli MF, Corrà U, Dendale P et al (2016) Challenges in secondary prevention after acute myocardial infarction: a call for action. Eur J Prev Cardiol 23:1994-2006

27. Turk-Adawi, Sarrafzadegan N, Grace SL. Global availability of cardiac rehabilitation. Nat Rev Cardiol.2014 October; 11 (10):586-596.

28. Duncan A, Natarajan MK, Schwalm JD. Assessing physician barriers to cardiac rehabilitation referral rates in a tertiary teaching centre. 2016. Can J Gen Int Med 11(1).

29. Cuenza LR, Gacrama EN, Tan K, Quito BJ, Ebba E (2016) Physician factors affecting cardiac rehabilitation referral among cardiac specialists: The Philippine Heart Center CRAVE study (Cardiac Rehabilitation Attitudes and Viewpoints on Engagement). J Clin Prev Cardiol 5:44-50

30. Grace SL, Grewal K, Stewart DE. Factors affecting cardiac rehabilitation referral by physician specialty. J Cardiopulm Rehabil Prev.2008 Jul-Aug; 28(4):248-52.

31. Ghisi LMG et al (2013) Physician factors affecting cardiac rehabilitation referral and patient enrollment: a systematic review. Clin Cardiol 36(6):323-335

32. Brown T et al. Predictors of cardiac rehabilitation referral in coronary artery disease patients. findings from the american heart association's get with the guidelines program. J Am Coll Cardiol.2009;54(6), august 2009

33. Barber K, Stommel M, Kroll J et al (2001) Cardiac rehabilitation for community-based patients with myocardial infarction: factors predicting discharge recommendation and participation. Clin Epidemiol 54:1025-1030

34. Pell J, Pell A, Morrison C, et al. Retrospective study of influence of devrivation on uptake of cardiac rehabilitation. BMJ.1996;313:267-268.

35. Ayanian JZ, Landrum MB, Gandagnolu, et al. Speciality of ambulatory care physicians and mortality among eldery patients after myocardial infarction. N Engl J Med.2002;347:1678-1686

36. Grace SL, Evindar A, Abramson BL, Stewart DE. Physician management preferences for cardiac patients: factors affecting referral to cardiac rehabilitation. Can J Cardiol. 2004 Sep; 20(11):1101-7.

37. de Melo GGL, Contractor A, Abhyankar M, Syed A, Grace SL (2018) Cardiac rehabilitation knowledge, awareness and practice among cardiologists in India. Indian Heart J 70(5):753-755

\section{Publisher's Note}

Springer Nature remains neutral with regard to jurisdictional claims in published maps and institutional affiliations. 